\title{
Transatlantica
}

Revue d'études américaines. American Studies Journal

Révolution

\section{« No more exit to route 66 »}

Lee Friedlander au Jeu de Paume (Novembre 2006)

\section{Marie Cordié Lévy}

\section{OpenEdition}

Journals

Édition électronique

URL : http://journals.openedition.org/transatlantica/1163

DOI : $10.4000 /$ transatlantica. 1163

ISSN : $1765-2766$

Éditeur

AFEA

\section{Référence électronique}

Marie Cordié Lévy, « « No more exit to route 66 »», Transatlantica [En ligne], 2 | 2006, mis en ligne le 14 mai 2008, consulté le 29 avril 2021. URL : http://journals.openedition.org/transatlantica/1163 ; DOI : https://doi.org/10.4000/transatlantica.1163

Ce document a été généré automatiquement le 29 avril 2021.

\section{(c) (i)}

Transatlantica - Revue d'études américaines est mis à disposition selon les termes de la licence Creative Commons Attribution - Pas d'Utilisation Commerciale - Pas de Modification 4.0 International. 


\title{
« No more exit to route $66 »$
}

\author{
Lee Friedlander au Jeu de Paume (Novembre 2006) \\ Marie Cordié Lévy
}

Compte rendu

1 La rétrospective proposée par Peter Galassi, conservateur en chef de la photographie au MOMA de l'œuvre de Lee Friedlander s'annonce comme le reflet de l'immense œuvre photographique de l'artiste exposé de nombreuses fois au MOMA $(1972,74,91)$.

2 Les 500 œuvres exposées au jeu de Paume proviennent toutes de livres publiés, tels «Self portrait» (1970), « the American Monument » (1976), « Nudes » (1991), « Flowers and Trees» (1981), au rez de chaussée, "Cherry Blossom Trees in Japan» (1986), «Sticks and Stones, Architectural America» (2004), «the Desert seen» (1996), «Staglieno Cemetary » (1993) et « Portraits » (1985), au premier étage.

3 Exposés sur un fond jaune, huit portraits en couleur, de jazzmen célèbres - Aretha Franklin, Miles Davis, John Coltrane, entre autres - que Lee fit à ses débuts pour les couvertures de disques Columbia, RCA ou Atlantic Record ouvrent l'exposition avec panache.

4 Les photos groupées par séries thématiques ou stylistiques de 4,6,8 ou plus suivent un déroulement chronologique souple le long des deux étages spacieux du «Jeu de Paume». Après une introduction rapide de $P$. Galassi, nous pouvons donc suivre l'évolution de la réflexion de L. Friedlander sur l'image : comment l'image est notre lien quotidien au monde (cf première photo de chambre d'hôtel avec télévision allumée), ou comment les présidents starifiés deviennent des icônes vernaculaires (portraits de Kennedy dans les vitrines des magasins), là où le monument commémoratif de l'histoire nationale sombre, lui, dans l'oubli, noyé par la vague consumériste des années soixante et les villes à expansion rapide (cf la série sur " the American monument »).

5 A la manière d'un Marcel Duchamp, Rrose Selavy, Lee Friedlander, dans la série des autoportraits, colle son ombre sur le col en fourrure d'une promeneuse (il est plus qu' « un intrus », comme il l'affirme, il est elle); s'identifie au désert, en posant son ombre décontractée sur les rochers pour y figurer ses seins, son estomac et ses viscères (canyon de Chelly 1983), s'auto-encense et s'auto-censure en se cachant le visage derrière une coupe trophée. Mais il se photographie aussi en caleçon, assis dans une 
chambre de motel, comme un homme à moitié mort attendant que la lumière (le bulbe de l'ampoule posé devant ses yeux dans l'autoportrait suivant) le fasse revivre. Les autoportraits, "extension périphérique de son travail, sont, dit-il, un petit rire nerveux, une langue tordue, une présence fascinante et dérangeante ».

Et c'est bien ce regard dérangeant que l'on retrouve dans une des premières photographies exposées, prise en Virginie en 1961: en renversant le signe "white only ", à lire à l'envers dans la vitre d'une vitrine, Lee Friedlander percute Leonard de Vinci et John Mc Laughlin afin de dénoncer la ségrégation, dénonciation qu'il reprendra de manière plus didactique dans la photographie prise au Texas en 67, « private, keep out ».

7 A la manière de Manuel Alvares Bravo dans ses «anges en camion» (1930), Lee Friedlander promène la photographie du pape dans les rues d'Italie.

8 Puis, troquant la distance respectueuse qu'imposait " the American Monument " pour une série de close-ups d'Américains en pleine "party », Lee Friedlander lance un clin d'œil transatlantique à William Klein, photographe de la vitalité et du plan rapproché. Enfin, il se tourne vers Charis, la femme que Weston photographia nue dans le désert de Californie pour la série de portrait de nus, faisant poser les corps à sa manière, décontractée et innocente. Mais le désert et le sable, la lumière de l'ouest ne sont pas au rendez-vous, les corps sont chez lui plus sombres, moins lumineux, plus fragmentés, comme s'il ne s'autorisait pas à leur rendre un hommage total. Dans la série des nus encore, Lee fait un petit clin d'œil à Kertesz, avec la belle danseuse sur le sofa, mais nue cette fois-ci.

9 C'est à partir du premier étage que Lee Friedlander quitte les maîtres pour prendre son envol - maitrise totale du médium, composition parfaite des paysages - et trouve enfin son souffle et sa puissance en choisissant l'aplat, la fin totale des lignes de fuites: le paysage devient une série de motifs qui envahit l'espace, étouffant le spectateur sous le cycle de ses répétitions. Le motif devient signe, comme dans la série des chiffres qui couvre le premier mur du premier étage, et on ne sait plus s'il influença là Edward Ruscha ( voir le « oof» de 1963) ou si ce fut l'inverse.

Rejouant le monde du paysage vernaculaire américain introduit par Walker Evans, avec ses maisons de bois blancs, ses voitures, ses poteaux, ses panneaux, ses cabines téléphoniques, il ajoute dans la série extraites de "Sticks and Stones » ces kilomètres de grillages qui cernent, encerclent, cadrent, défendent des propriétés minables auxquelles s'accrochent des herbes grimpantes tenaces qui bordent la fameuse route 66. Saturant l'espace de signes, de simulacres et de faux semblants, il crée un effet d'obstruction, nous introduit dans un univers vide, où les contacts humains deviennent des escapades aussi fulgurantes que l'étroitesse des échancrures, quand ils ne restent pas à l'état de regards croisés à travers les reflets des rétroviseurs ou des vitres des voitures, comme dans les jeux de miroirs présents dans les photographies du rez-dechaussée. Mais ici l'image s'investit en profondeur dans le multiforme, l'éclaté, le polyvalent alors qu'au rez de chaussée elle restait encore en surface.

11 Enfin, il reprend et renverse le mythe de l'ouest américain, univers imposant de splendeur et d'harmonie introduit par Ansel Adams pour en faire un monde pétrifié de branches mortes (d'où l'impression de all-over à la Pollock) comme si justement la nature ayant perdu sa valeur paradisiaque se refusait à vivre, pour n'être plus que le reflet artificiel de sa lointaine existence. 
12 Du regard distrait et nerveux, inquiet du début, Lee Friedlander est passé à une parfaite maîtrise du décalage photographique, cinglant l'idéologie expansionniste, ramenant la communication des êtres à ce qu'elle est, un fil ténu de téléphone, une ligne blanche qui parcourt le macadam. Lee Friedlander nous oblige à regarder le monde tel qu'il est : un monde indifférent à l'homme, un monde surcadré, saturé, mais un monde vide et mort.

13 Après la superposition jazzy, kerouacienne, des reflets et des impressions du début, c'est par ce travail de déconstruction photographique du monde que Lee Friedlander nous intéresse le plus aujourd'hui.

14 La dernière salle située un peu à l'écart du reste de l'exposition offre une série de portraits que Lee Friedlander fit de ses proches, de sa femme, son fils, son petit-fils, ses amis. Tout en pudeur et en retenue, il y fait preuve d'une grande sensibilité et délicatesse d'esprit, et donne l'impression que maintenant il s'est retiré de la grande bataille du monde pour profiter enfin des siens.

15 Cette rétrospective ambitieuse manque peut-être d'un travail écrit d'accompagnement des différentes séries afin de mener le visiteur vers une appréciation plus profonde du travail de l'artiste (chaque titre d'ouvrage pourrait être accompagné de quelques éléments explicatifs replaçant le travail de l'artiste au sein de sa propre problématique et des influences culturelles et civilisationnelles du moment). Nous regrettons aussi que les dates accompagnant les œuvres soient si minuscules.

Three questions to Peter Galassi (October 30th, 2006).

16 Peter Galassi, conservateur en chef de la photographie au MOMA a accepté de répondre à ces trois questions alors que je visitais le MOMA avec mes étudiants d'histoire de la photographie.

17 1/MCL : Compared to other artists who used their shadow in their photographs or paintings (Atget, Kertesz, Stieglitz or Picasso and Jasper Johns), do you think Friedlander's use of his shadow is different from theirs? Does he wish to establish another relation with the public? If yes, what kind of relationship?

18 P.G. : His work belongs to the 60's. It is a new style, an art of photographic mistakes. The shadows are not done on purpose, as in Atget or Stieglitz. They are different, they are an interesting game, they show the malleability and changeability of personality. He didnt stop at the reflection. The shadows have all kinds of meanings.

19 2/ MCL : When considering the group of pictures with the petrified trees, would you say Friedlander's approach to the landscape has been influenced by Pollock's all over technique? Can this vision of the American landscape be considered as schizophrenic? P.G. :The trees are not influenced by Pollock. Friedlander was interested in literature. His work is interesting to me because we think of Pollock. His work has been interpreted in different ways. Kitaj said «Friedlander reminds me of Bonnard». His trees are very American, and relate to the landscape tradition in photography. He used the traditional way and made it his own. His work relates to the big wild nature.

21 3/ MCL : The layout of the exhibition on Friedlander is outstanding. You chose to group the pictures according to a formalistic aprroach, what Rimbault called " correspondance ». Is there a progression from one group to the next? Don't you think this formalistic panoramic approach prevents us from perceiving the sensitive meaning behind each photograph? 
P.G: The layout comes from his work, this is how he worked, when he sees something that works, he sees how far he can go, it is in the nature of his work to work in the family of his work. The layout of the exhibition goes all the way back to the beginning, it is the proximity of the photographs that makes them relevant.

INDEX

Thèmes : Trans'Arts

\section{AUTEUR}

\section{MARIE CORDIÉ LÉVY}

Professeur d'anglais et d'histoire de la photographie, Lycée Jules Ferry, Paris $9^{\text {e }}$. 